\title{
Síndrome da Hipoventilação da Obesidade: manejo terapêutico e principais repercussões clínicas
}

Obesity Hypoventilation Syndrome: therapeutic management and its clinical repercussions

\author{
Síndrome de Hipoventilación por Obesidad: manejo terapéutico y principales \\ repercusiones clínicas
}

Juliana Costa Ribeiro1*, lasmin Marques Ferreira1, Marcela Alves Gusmão1, Karen Vitoria Martins Oliveira Reis ${ }^{2}$, Caroline Domingues Zwicker ${ }^{3}$, Juliana Corá da Silva ${ }^{4}$, Ana Clara Mendonça de Carvalho ${ }^{5}$, Thales Miranda Sales ${ }^{2}$.

\section{RESUMO}

Objetivo: Discorrer sobre seus impactos clínicos e de comorbidades associadas, abordando também, medidas terapêuticas e suas eficácias. Revisão bibliográfica: A SHO é uma condição crônica dada pela associação de fatores como hipercapnia, alterações respiratórias e obesidade. Juntamente a SHO podem surgir outros distúrbios, como AOS e alterações cardiovasculares, as quais se correlacionam com desfechos desfavoráveis. O tratamento de escolha inicial da SHO é o CPAP, sendo indispensável uma abordagem multidisciplinar sobre a melhora da higiene do sono e mudanças de estilo de vida, medidas essas, consideradas primordiais. Considerações finais: Por meio de uma análise sem sistematização e comparação com outras técnicas disponíveis, constatou-se que o tratamento de escolha é feito com o CPAP, devido a redução do Índice de Apneia-Hipopneia (IAH) na maioria dos pacientes, não sendo necessária a associação com outras medidas terapêuticas. Terapias como a traqueostomia e flebotomia devem ser realizadas somente se necessário. Além disso, devem ser evitadas medicações como medroxiprogesterona e acetazolamida e o uso excessivo de diuréticos de alça e sedativos/hipnóticos.

Palavras-chave: Síndrome hipoventilação por obesidade, Apnéia Obstrutiva do sono, Polissonografia, Ventilação não invasiva, Síndrome de Pickwick.

\begin{abstract}
Objective: Discuss their clinical impacts and associated comorbidities, also addressing therapeutic measures and their effectiveness. Literature review: OHS is a chronic condition given by the association of factors such as hypercapnia, respiratory changes and obesity. Along with OHS, other disorders may arise, such as OSA and cardiovascular alterations, which are correlated with unfavorable outcomes. The treatment of choice for OHSS is CPAP, and a multidisciplinary approach to improving sleep hygiene and lifestyle changes is essential, which are considered essential measures. Final considerations: Through an analysis without systematization and comparison with other available techniques, it was found that the treatment of choice is made with CPAP, due to the reduction in the Apnea-Hypopnea Index (AHI) in most patients, not being association with other therapeutic measures is necessary. Therapies such as tracheostomy and phlebotomy should be performed only if necessary. In addition, medications such as medroxyprogesterone and acetazolamide and excessive use of loop diuretics and sedatives/hypnotics should be avoided.
\end{abstract}

Key words: Obesity hypoventilation syndrome, Obstructive sleep apnea, Polysomnography, Non-invasive ventilation, Pickwick syndrome.

\footnotetext{
1 Universidade de Itaúna, Itaúna - MG. *E-mail: julianaribeirocosta@hotmail.com

2 Centro Universitário de Caratinga, Caratinga - MG.

${ }^{3}$ Universidade de Araraquara, Araraquara - SP.

${ }^{4}$ Universidade Estadual de Maringá, Maringá - PR.

${ }^{5}$ Universidade de Fortaleza, Fortaleza - CE.
}

SUBMETIDO EM: 5/2021 | ACEITO EM: 5/2021 ～PUBLICADO EM: 6/2021 


\section{RESUMEN}

Objetivo: Discutir sus impactos clínicos y comorbilidades asociadas, abordando también las medidas terapéuticas y su efectividad. Revisión de la literatura: El SHO es una condición crónica dada por la asociación de factores como hipercapnia, alteraciones respiratorias y obesidad. Junto con el SHO pueden surgir otros trastornos, como la AOS y las alteraciones cardiovasculares, que se correlacionan con desenlaces desfavorables. El tratamiento de elección para el SHO es la CPAP, y es fundamental un enfoque multidisciplinario para mejorar la higiene del sueño y los cambios en el estilo de vida, que se consideran medidas fundamentales. Consideraciones finales: A través de un análisis sin sistematización y comparación con otras técnicas disponibles, se encontró que el tratamiento de elección se realiza con CPAP, debido a la reducción del Índice de Apnea-Hipopnea (IAH) en la mayoría de los pacientes, no estando asociado con otros Es necesario tomar medidas terapéuticas. Las terapias como la traqueotomía y la flebotomía deben realizarse solo si es necesario. Además, se deben evitar medicamentos como medroxiprogesterona y acetazolamida y el uso excesivo de diuréticos de asa y sedantes / hipnóticos.

Palabras clave: Síndrome de hipoventilación por obesidad, Apnea obstructiva del sueño, Polisomnografía, Ventilación no invasiva, Síndrome de Pickwick.

\section{INTRODUÇÃO}

A Síndrome de Hipoventilação da Obesidade (SHO) é caracterizada pela combinação de fatores como a obesidade (índice de massa corporal $\geq 30 \mathrm{~kg} / \mathrm{m}^{2}$ ), hipercapnia diurna (concentração parcial de dióxido de carbono arterial $\geq 45 \mathrm{mmHg}$ ) e Distúrbios Respiratórios do Sono (DRS), associado a uma insuficiência respiratória com hipercapnia crônica, sendo que se faz necessário descartar outros distúrbios que possam também cursar com hipoventilação alveolar (MASA JF, et al., 2019).

Considerada uma desordem respiratória grave relacionada com a obesidade, a SHO é uma condição crônica, acompanhada em mais de $90 \%$ dos casos com Apneia Obstrutiva do Sono (AOS), podendo repercutir ainda com uma diminuição das atividades de vida diária dos pacientes associada a taxas mais altas de mortalidade, insuficiência cardíaca crônica, hipertensão pulmonar e aumento dos números de hospitalizações devido ao quadro de hipercapnia aguda (TERREROS FJG, et al., 2019).

A SHO possui um diagnóstico de exclusão, necessitando que outras causas de hipoventilação sejam descartadas inicialmente. É rotineiramente diagnosticada após um quadro de insuficiência respiratória hipercápnica aguda ou por sintomas crônicos de DRS (MASA JF, et al., 2018). Apesar de possuir alta prevalência e relevância clínica, ainda é amplamente subdiagnosticada e é frequentemente reconhecida apenas nos estágios finais da doença, com a presença de comorbidades cardiovasculares e metabólicas (ATHAYDE RA, et al., 2018; TERREROS FJG, et al., 2019).

O intuito do tratamento é controlar as anormalidades causadas pela síndrome, normalizar o distúrbio respiratório e melhorar as trocas gasosas, buscando uma perda ponderal, uma vez que esta comorbidade afeta significativamente o seguimento da SHO (ATHAYDE RA, et al., 2018; PIPER A, et al., 2020).

Além disso, uma das principais abordagens em pacientes com SHO é o uso de ventilação mecânica no modo pressão positiva na via aérea (CPAP). A finalidade é promover uma normalização da PaCO2; prevenir dessaturações; controlar a hipertensão pulmonar e a cor pulmonale, além de aliviar a hipersonia. De modo que, para o tratamento da SHO, é preciso buscar associação de estratégias terapêuticas (ATHAYDE RA, et al., 2018; MASA JF, et al., 2019).

O presente artigo teve como objetivo realizar uma revisão de literatura sobre a SHO em conjunto com as comorbidades associadas, compreendendo os impactos clínicos dessa síndrome. Pretendeu-se também, abranger os tratamentos já descritos e assim, evidenciar a efetividade da terapia com pressão positiva, além de esclarecer sobre abordagens que se mostraram ineficazes. 


\section{REVISÃO BIBLIOGRÁFICA}

A SHO define-se através da combinação de fatores como hipercapnia, alterações respiratórias e obesidade. Devido a isso, os distúrbios do sono encontram-se presentes, sendo que a maioria desses pacientes possuem apneia obstrutiva do sono. Há também um número crescente da taxa de obesidade global, juntamente com os custos adquiridos através disso. Levando-se em conta a importância da saúde, tem-se maior interesse em desmembrar essa síndrome e os tipos de tratamentos que otimizam os resultados e reduzem os gastos. O mecanismo fisiopatológico pelo qual a obesidade leva a hipoventilação é complexo e não completamente compreendido. Vários mecanismos têm sido propostos na patogênese da SHO, incluindo alterações mecânicas respiratórias secundárias à obesidade, hipercapnia aguda devido aos eventos obstrutivos durante 0 sono, hipoventilação central secundária a resistência à leptina e uma resposta compensatória alterada à hipoventilação crônica durante o sono (IFTIKHAR IH e ROLAND J, 2018; PIPER A, et al., 2020).

Dentre as combinações de fatores da SHO há um quadro clínico comum de hipoxemia, hipercapnia e alterações neuro-humorais. A obesidade é uma das principais causas da doença, sendo as modificações do padrão respiratório relacionados não somente a quantidade de gordura, mas também a estruturação do tecido adiposo pelo corpo. Distribuições centrípeto e periférico se diferem, por exemplo, sendo a primeira maior influente sobre a mecânica ventilatória e com maior predominância em pacientes com SHO. Além disso, o diafragma, órgão coparticipante da respiração, é influenciado pela deposição da gordura abdominal causando danos à função pulmonar, e, inclusive, provocando adelgaçamento desse músculo (ATHAYDE RA, et al., 2018).

O alto índice do tecido adiposo faz com que o trabalho respiratório intensifique, provocando uma resistência pulmonar aumentada e uma complacência pulmonar diminuída. A troca gasosa também é comprometida através da respiração em menor volume corrente e elevação da frequência respiratória. Dessa forma, resulta em um quadro clínico comum de hipoxemia, apresentando, concomitantemente, hipertensão pulmonar secundária à hipóxia. Isso faz com que $\mathrm{CO} 2$ seja elevado e aumente a absorção de bicarbonato. Há a diminuição do $\mathrm{pH}$ do líquido cefalorraquidiano e consequente hipercapnia ventilatória (ATHAYDE RAB, et al., 2018; IFTIKHAR IH e ROLAND J, 2018).

A leptina, proteína produzida pelo tecido adiposo, possui contribuição em relação a obesidade, hipercapnia crônica e ao controle ventilatório alterado na síndrome. Tal hormônio possui níveis elevados em obesos devido à maior presença de adipócitos. A função no hipotálamo é inibir o apetite. Com o excesso de peso, há mais adipócitos e, por consequência, maiores níveis circulantes de leptina, o que também se apresenta sendo um estimulante a resposta ventilatória, semelhante a um fator protetor. Entretanto, devido a obesidade, ocorre uma resistência à leptina, acarretando na diminuição da resposta ventilatória ao CO2. Essa alteração do controle metabólico leva a alterações cardiometabólicas e influencia diretamente no sono (IFTIKHAR IH e ROLAND J, 2018).

\section{Comorbidades e repercussões clínicas}

Juntamente a SHO podem surgir outros distúrbios, como a Apneia Obstrutiva do Sono (AOS) e também alterações no sistema cardiovascular, sendo essas comorbidades foco de atenção ao longo dos anos devido aos desfechos desfavoráveis que podem apresentar. Esses fatores são considerados de risco para o desenvolvimento de doenças cardiovasculares e podem se relacionar direta ou indiretamente com ataques isquêmicos transitórios, acidente vascular encefálico e hemorragias intracerebrais (ATHAYDE RA, et al., 2018; PARRA O e ARBOIX A, 2016).

Pacientes com AOS apresentam quadros de hipoxemia noturna repetitiva, a qual apresenta correlação com diferentes mecanismos que podem ajudar a explicar o desenvolvimento de doenças cardíacas e vasculares associadas a essa condição. A hipoxemia é responsável pela ativação de resposta inflamatória e metabólica, além de desencadear resposta neural, trombótica e humoral. Esses mecanismos podem ser ativados mesmo naqueles pacientes que não possuíam um distúrbio cardiovascular prévio, o que sinaliza que 
o próprio distúrbio do sono em associação com a síndrome de obesidade podem ser fatores desencadeadores ou aceleradores de algumas doenças cardiovasculares (SHAMSUZZAMAN ASM, et al., 2003).

A apneia obstrutiva do sono tem consequências importantes. Muitos indivíduos que desenvolvem essa condição possuem um sono fragmentado, pois precisam acordar para respirar devido a interrupções noturnas que a doença causa. As consequências desse sono fragmentado repercutem de diferentes formas reduzindo a qualidade de vida do indivíduo, além de comprometer sua função cognitiva e capacidade de concentração, podendo ele se tornar mais sonolento durante o dia e colocando-o em risco de acidentes e demais complicações. Outros fatores que possam explicar as consequências da apneia obstrutiva do sono sobre 0 sistema cardiovascular são a liberação de hormônios plasmáticos, como as catecolaminas, através de períodos de hipoxemia e hipercapnia que levam ao despertar do sono (JAISWAL SJ, et al., 2017).

Diferentes estudos de revisão sistemática buscaram explicar a relação entre a apneia obstrutiva do sono e o acometimento do sistema cardiovascular. Foram encontrados diferentes mecanismos que podem explicar porque indivíduos com AOS são mais susceptíveis a desenvolverem quadros hipertensivos, bem como acidente vascular encefálico e outras doenças cerebrovasculares. Alguns mecanismos identificados foram a desregulação do metabolismo endócrino, estresse oxidativo, ativação do sistema nervoso simpático e da cascata de coagulação e também disfunções do endotélio dos vasos. Foi observado que pacientes que possuem histórico de AVE com causa a esclarecer apresentavam maior incidência de distúrbios respiratórios do sono, como a apneia obstrutiva, confirmando então que essas condições influenciam, de maneiras ainda a serem esclarecidas totalmente, a ocorrência desses eventos cardiovasculares. Além disso, foi também observado que pacientes com histórico de AVE e que desenvolveram apneia obstrutiva do sono possuíam risco aumentado de morte em relação àqueles sem o distúrbio respiratório do sono (PARRA O e ARBOIX A, 2016).

Além dos distúrbios vasculares, pacientes com apneia obstrutiva do sono podem ter maiores riscos de ter distúrbios de condução cardíaca e desenvolverem quadros de arritmias cardíacas, se comparados àqueles sem AOS. Em um estudo de caso controle, foi evidenciado que a fibrilação atrial (FA) foi mais frequentemente observada em indivíduos com AOS e que esses pacientes tiveram maior risco de desenvolverem acidentes vasculares encefálicos do que aqueles sem AOS. Em adição a isso, evidências mostraram que a presença da fibrilação atrial como doença de base pode desencadear piora nessas outras condições e comorbidades associadas, como a AOS e demais doenças como miopatias e hipertensão arterial sistêmica (JEHAN S, et al., 2018).

Existem algumas modalidades de tratamento que buscam melhorar as consequências do AVE em pacientes que possuem a apneia obstrutiva do sono associada. O uso do CPAP, por exemplo, é responsável por melhorar significativamente os efeitos do acidente isquêmico, como deficiências físicas e intelectuais que possam ter surgido. Esse tratamento ainda é um processo desafiador, que necessita de outras abordagens complementares para garantir seu sucesso. De forma a superar e minimizar a ocorrência e as consequências da AOS é preciso controlar fatores que estão intrinsecamente relacionados a gravidade do quadro, como a obesidade. Apenas através de uma abordagem multidisciplinar será possível reduzir consequências graves como AVE, hipertensão e demais doenças cardiovasculares. (JEHAN S, et al., 2018).

\section{Manejo e tratamentos}

Não se sabe ao certo como a obesidade explica fisiopatologia da síndrome por completa, mas tem-se conhecimento que sua presença é a grande responsável pelos quadros respiratórios observados nesses pacientes obesos. Diante disso, atenta-se para o fato de que o tratamento não deve envolver apenas a redução de sequelas e desordens respiratórias, mas sim utilizar de medidas e técnicas multidisciplinares que busquem estimular mudança no estilo de vida de forma a tratar a obesidade, que segue sendo preditor de surgimento e de agravo da condição (PIPER A, et al., 2020).

O tratamento de escolha inicial a SHO que se encontra estável é o uso da pressão positiva das vias aéreas, a CPAP. Seu uso é capaz de aumentar a ventilação central e reduzir a resistência das vias aéreas superiores. O índice de apneia-hipopneia (soma de todas as apenas e hipopneias durante uma hora de sono) é reduzido 
para menos de 10 eventos por hora no sono de muitos pacientes. Em decorrência disso, o número de despertares durante a noite diminui, reduzindo significativamente as consequências de um sono fragmentado e prejudicial para a resolução das atividades de vida diárias (ATHAYDE RAB, et al., 2018; FARIA AC e CHIBANTE F, 2016).

Uma abordagem multidisciplinar é indispensável. De início, é considerado primordial a melhora da higiene do sono e a adequação do paciente a um estilo de vida mais saudável, que irá incluir não apenas a perda de peso. Os profissionais responsáveis devem pesquisar o uso de medicações que podem se relacionar com a atividade muscular respiratória, como por exemplo benzodiazepínicos, narcóticos e barbitúricos. É preciso orientar o paciente a respeito da importância de reduzir ou interromper o consumo de álcool e tabagismo, além de estimular a prática de atividades físicas regulares. (ANDRADE FMC e PEDROSA RP, 2016)

A perda de peso pode ser obtida por meio de acompanhamento profissional com nutricionista e professores de educação física. $\mathrm{O}$ emagrecimento está associado a grande melhoria dos parâmetros respiratórios, sendo que uma perda de cerca de 10kg é capaz de reduzir a pressão parcial de CO2 durante o dia e aumentar a capacidade vital máxima. (OLSON AL e ZWILLICH C, 2005)

Muitos pacientes possuem indicação de realizar cirurgia bariátrica, em virtude dos valores elevados de massa corpórea. A National Institutes of Health possui diferentes designações da indicação cirúrgica para pacientes com ou sem comorbidades. Caso o paciente possua um $\mathrm{IMC}>35 \mathrm{~kg} / \mathrm{m} 2$ e síndrome da hipoventilacao-obesidade ou com $I M C>40 \mathrm{~kg} / \mathrm{m} 2$ é indicado o tratamento cirúrgico primariamente, com adoção de medidas dietéticas/nutricionais e de mudança de qualidade de vida como manutenção (OLSON AL e ZWILLICH C, 2005).

Um fato bastante observado é que pacientes com obesidade mórbida muitas vezes não conseguem sustentar a perda de peso, seja ela obtida cirurgicamente através da cirurgia bariátrica bem como pela adoção de um estilo de vida diferente, sendo isso explicado por diferentes mecanismos biológicos associados a estagnação do peso. Como terapia adjuvante pode ser associada a farmacoterapia vida do aumentar a perda de peso. De uma forma geral, a perda de peso varia entre os pacientes, mas se alcançado valores satisfatórios pode ser responsável pela melhora dos distúrbios respiratórios do sono e demais comorbidades (ATHAYDE RAB, et al., 2018; WOLFE BM, et al., 2016).

A perda de peso é altamente sugerida para pacientes com AOS e SHO devido aos seus benefícios para a redução das comorbidades, melhoria de qualidade de vida e redução dos índices de morbimortalidade. A perda de peso é responsável pela redução do risco de doenças cardiovasculares graves e que produzem sequelas permanentes. Além disso, a redução do IMC relaciona-se a menor ocorrência de quadros metabólicos, inflamatórios e respiratórios, devendo ser indicada a todos os pacientes obesos com comorbidades associadas. (WOLFE BM, et al., 2016).

\section{Tratamentos ineficientes}

A oximetria de pulso, quando utilizada de modo isolado na SHO detecta apenas a dessaturação da oxihemoglobina e não a hipercapnia, levando a um diagnóstico perdido, pois os pacientes são tratados erroneamente em monoterapia de oxigênio suplementar, o que não suprime a hipoventilação da SHO. Em casos de pacientes hemodinamicamente estáveis sem trabalho respiratório excessivo, deve-se utilizar gasometria arterial para confirmar a presença de hipercapnia diurna (OLSON AL, et al., 2005).

Devido aos riscos e complicações associados ao procedimento de traqueostomia, evita-se realizá-la em pacientes obesos, sendo apenas assimilada em casos resistentes à ventilação não invasiva (VNI). Além disso, na farmacoterapia não é recomendado o uso de medroxiprogesterona e acetazolamida para ampliar a resposta ventilatória, pois já foram tentadas, mas não apresentaram êxito. Já medicamentos sedativos/hipnóticos com seus usos excessivos reduzem a resposta ventilatória e aumentam a colapsibilidade das vias aéreas, prejudicando ainda mais a hipoventilação no SHO (ATHAYDE RAB, et al., 2018).

Outro cuidado é com o uso excessivo de diurético de alça (furosemida), que pode piorar a hipoventilação diurna e a hipoxemia, devido ao agravamento da alcalose metabólica. Indica-se o uso cauteloso na menor 
dose plausível para diminuir o impacto eletrolítico e ácido-metabólico de diuréticos. A furosemida é utilizada para obter um estado euvolêmico, porém no caso de SHO os pacientes são acometidos por edemas devido à cor pulmonale (MANTHOUS CA e MOKHIESI B, 2015; ATHAYDE RAB, et al., 2018).

Na literatura há dados insuficientes sobre os efeitos adversos e resultados de longo prazo sobre o uso da terapia de progesterona na frequência de apneia ou sintomas de sonolência. O que se sabe para SHO é que a medroxiprogesterona demonstra um aumento na quimiossensibilidade hipercápnica e melhora de ventilação, mas são resultados insuficiente para tratar SHO (OLSON AL, et al., 2005).

A eritrocitose está presente em pacientes com SHO secundariamente, com o intuito de gerar uma resposta fisiológica do corpo à hipóxia. Para tratá-la pode ser utilizado flebotomia, porém não há dados suficientes que comprovem a eficácia em SHO, sendo a utilização com flebotomia uma terapêutica raramente admitida para tal síndrome (MOKHLESI B e TULAIMAT A, 2007; HEINEMAN F, et al., 2007). Portanto, profissionais da saúde devem avaliar pacientes com suspeita de SHO através de características individuais, comorbidades e fatores de risco associados para uma terapêutica eficiente (TERREROS FJG, et al., 2019).

\section{CONSIDERAÇÕES FINAIS}

Este estudo contempla uma análise sem sistematização e embora não tenha sido feita uma comparação com todas as técnicas disponíveis, observa-se que o tratamento com CPAP é o de escolha, pois pode reduzir o Índice de Apneia-Hipopneia (IAH) na maioria dos pacientes. Entretanto, é necessário associar outras medidas terapêuticas para promover a melhora dos parâmetros respiratórios, como a perda de peso e realização de atividades físicas. Algumas terapias devem ser usadas somente se necessário, como a traqueostomia e flebotomia; e outras medicações devem ser evitadas, como a medroxiprogesterona e acetazolamida e o uso excessivo de diurético de alça e sedativos/hipnóticos.

\section{REFERÊNCIAS}

1. ANDRADE FMD, PEDROSA RP. The role of physical exercise in obstructive sleep apnea. J Bras Pneumol, 2016; 42(6): 457-464.

2. ATHAYDE RAB, et al. Síndrome de obesidade-hipoventilação: uma revisão atual. J Bras Pneumol, 2018;44(6):510518.

3. BAHAMMAM AS. Prevalence, clinical characteristics, and predictors of obesity hypoventilation syndrome in a large sample of Saudi patients with obstructive sleep apnea. Saudi Med J, 2015; 36(2): 181-189.

4. BOREL JC, et al. Obesity hypoventilation syndrome: From sleep-disordered breathing to systemic comorbidities and the need to offer combined treatment strategies. Respirology, 2012; 17(4): 601-610.

5. CHIRINOS JA, et al. CPAP, Weight Loss, or Both for Obstructive Sleep Apnea. N Engl J Med, 2014; 370(24): 2265 2275.

6. DONG Z, et al. Weight loss surgery for obstructive sleep apnoea with obesity in adults: a systematic review and metaanalysis protocol. BMJ Open, 2018; 8(8): e020876.

7. FARIA AC, CHIBANTE F. Pressão positiva nas vias aéreas (CPAP) no tratamento da apneia obstrutiva do sono. Revista HUPE, 2016; 15(1): 75-81.

8. HEINEMANN F, et al. Non-invasive positive pressure ventilation improves lung volumes in the obesity hypoventilation syndrome. Respir Med, 2007; 101(6): 1229-1235.

9. HUANG JF, et al. Obesity hypoventilation syndrome and severe COVID-19. Metabolism, 2020; 108:154249.

10. IFTIKHAR IH, ROLAND J. Obesity Hypoventilation Syndrome. Clin Chest Med, 2018; 39(2): 427-436.

11. JAISWAL SJ, et al. Raising awareness about sleep disorders. Lung India, 2017; 34(3): 262-268.

12. JEHAN S, et al. Obstructive sleep apnea and stroke. Sleep Med Disord. 2018; 2(5): 120-125.

13. LIU C, et al. The relationship between obstructive sleep apnea and obesity hypoventilation syndrome: a systematic review and meta-analysis. Oncotarget, 2017; 8(54): 93168-93178.

14. LLANO LAP, et al. Short-term and long-term effects of nasal intermittent positive pressure ventilation in patients with obesity-hypoventilation syndrome. Chest, 2005; 128(2): 587-594.

15. MANTHOUS CA, MOKHLESI B. Avoiding Management Errors in Patients with Obesity Hypoventilation Syndrome. Ann Am Thorac Soc, 2016; 13(1): 109-114.

16. MASA JF, et al. Long-term clinical effectiveness of continuous positive airway pressure therapy versus non-invasive ventilation therapy in patients with obesity hypoventilation syndrome: a multicentre, open-label, randomised controlled trial. Lancet, 2019; 393(10182):1721-1732. 
17. MASA JF, et al. Long-term Noninvasive Ventilation in Obesity Hypoventilation Syndrome without Severe Obstructive Sleep Apnea. Chest, 2020; 158(3):1176-1186.

18. MASA JF, et al. Obesity hypoventilation syndrome. Eur Respir Rev, 2019; 28(151): 180097.

19. MOKHLESI B, TULAIMAT A. Recent Advances in Obesity Hypoventilation Syndrome. Chest, 2007; 132(4): 13221336.

20. NOWBAR S, et al. Obesity-associated hypoventilation in hospitalized patients: prevalence, effects, and outcome. Am J Med, 2004; 116(1): 1-7.

21. OLSON AL, ZWILLICH C. The obesity hypoventilation syndrome. Am J Med, 2005; 118(9): 948-956.

22. PARRA O, ARBOIX A. Stroke and sleep-disordered breathing: A relationship under construction. World J Clin Cases, 2016; 4(2): 33-37.

23. PIPER A, et al. Positive airway pressure in obesity hypoventilation syndrome: is it worth it?. Thorax, 2020; 75(6):439440.

24. SHAMSUZZAMAN ASM, et al. Obstructive Sleep Apnea: Implications for Cardiac and Vascular Disease. JAMA, 2003; 290(14): 1906-1914.

25. SIVAM S, et al. Obesity Hypoventilation Syndrome: Early Detection of Nocturnal-Only Hypercapnia in an Obese Population. J Clin Sleep Med, 2018; 14(09): 1477-1484.

26. TERREROS FJG, et al. Clinical Practice Guideline Summary for Clinicians: Evaluation and Management of Obesity Hypoventilation Syndrome. Ann Am Thorac Soc, 2020; 17(1):11-15.

27. TRAKADA GP, et al. Prevalence and clinical characteristics of obesity hypoventilation syndrome among individuals reporting sleep-related breathing symptoms in northern Greece. Sleep Breath, 2010; 14(4): 381-386.

28. TURNBULL CD, et al. Relationships between MRI fat distributions and sleep apnea and obesity hypoventilation syndrome in very obese patients. Sleep Breath, 2018; 22(3): 673-681.

29. WOLFE BM, et al. Treatment of Obesity: Weight Loss and Bariatric Surgery. Circ Res, 2016; 118(11): $1844-1855$. 